
\title{
$\begin{array}{ll}\text { Research Square } & \text { Preprints are preliminary reports that have not undergone peer review. } \\ \text { They should not be considered conclusive, used to inform clinical practice, } \\ \text { or referenced by the media as validated information. }\end{array}$
}

\section{Pregnant Women's Health-related Behavior Changes and Psychological Status After the Peak of COVID- 19 Outbreak in China: A Cross-sectional Study}

\section{Ruixue Tian}

Wuhan University School of Health Sciences

\section{Xu Zhang}

Wuhan University School of Health Sciences

\section{Xiaoli Chen}

Wuhan University School of Health Sciences

Che Deng

Wuhan University School of Health Sciences

\section{Zhijie Zou}

Wuhan University School of Health Sciences

Yanqun Liu ( $\nabla$ liuyanqun1984@163.com )

Wuhan University School of Health Sciences

\section{Research article}

Keywords: COVID-19, pregnant women, anxiety, depression, stress, psychological status, health-related behavior

Posted Date: September 3rd, 2020

DOI: https://doi.org/10.21203/rs.3.rs-67761/v1

License: (c) (1) This work is licensed under a Creative Commons Attribution 4.0 International License. Read Full License 


\section{Abstract}

Background: Little is known about the relationship between health-related behavior and psychological status of pregnant women during the COVID-19 outbreak. We aimed to describe the health-related behavior changes and psychological status of Chinese pregnant women, and to explore the relationship between pregnant women's characteristics, health-related behavior and different psychological status following the peak of COVID-19 outbreak.

Methods: We conducted an online survey through social media (WeChat) from March 14 to April 7. A selfdesigned questionnaire was used to investigate Chinese pregnant women's health-related behavior changes. Generalized Anxiety Disorder scale, Edinburgh Postnatal Depression Scale and Perceived Stress Scale were used to assess anxiety, depression and stress among pregnant women. Wilcoxon signed-rank test was used to compare health-related behavior changes before and during the COVID-19 outbreak and Spearman's rank correlation analysis or logistic regression analysis were used to explore the relationships between variables.

Results: A total of 113 pregnant women were included in this study. Most pregnant women wore masks (98.2\%), changed location or tried to stay away from those who seem to have a cold or fever $(99.1 \%)$ and washed hands more often than before (96.5\%). Pregnant women washed hands with soap or hand sanitizer more often and went out less often than before the outbreak. There were $41.6 \%$ pregnant women who didn't have antenatal visits or weren't on time for antenatal visits. Majority (77.9\%) pregnant women sought help when feeling panic or anxiety, whereas few turned to psychological workers. The rates of pregnant women with anxiety, depression and health risk stress were $64.6 \%, 58.4 \%$ and $54.9 \%$, respectively. Analysis of relationship between variables showed that household income, gestational age, pregnancy complications, knowledge about COVID-19 and maternal and child protection, perception of the likelihood of infection, the frequency of going out, prenatal check-up behavior, help-seeking behavior were significantly associated or correlated with psychological status.

Conclusions: Attention should be paid to the mental health of pregnant women with specific healthrelated behaviors changes. Screening for mental health problems and psychological interventions among pregnant women are needed.

\section{Background}

At the end of 2019, a pneumonia of unknown etiology (later named coronavirus disease 2019 (COVID-19) by the World Health Organization) broke out in Wuhan, Hubei Province [1], and quickly spread throughout China and even other countries [2]. In response to the COVID-19 epidemic, China has taken a series of prevention and control measures, such as travel restrictions, building Fangcang shelter hospitals and pairing assistance [3], which have brought the epidemic under control. From March 11 to 17, the daily number of new local confirmed cases remained in single digits, and the peak of the epidemic in China has passed as a whole [4]. Despite the gradual resumption of work and production in China, domestic 
sporadic cases and imported cases from abroad have been reported [4]. Preventing a rebound and preventing imports from abroad are essential.

During the initial stage or peak of the COVID-19 outbreak, studies from China [5, 6], Turkey [7], Italy [8], Canada [9], and Israel [10] have found that the COVID-19 had adverse psychological impact on women during pregnancy, such as increasing anxiety and depression symptoms. Several studies reported the behavior responses of pregnant women during the COVID-19 epidemic, such as wearing masks, washing hands more frequently and stocking up behaviors [11-13]. Protective measures were adopted by most pregnant women in China [12], whereas practice of preventive measures was poor among pregnant women in Nigeria [13]. Population's mental health and behavior are both important in preventing infectious diseases [14]. Psychological distress of pregnant women can also affect their own health and the physiological, cognitive, and psychological development of their offspring [15]. However, little is known about the health-related behavior changes and psychological status of pregnant women after the peak of the COVID-19 outbreak. The relationship between pregnant women's health-related behavior and psychological status during the outbreak is also unclear.

The aim of this study was to describe the health-related behavior changes and psychological status of pregnant women after the peak of COVID-19 outbreak. In addition, the relationship between pregnant women's characteristics, health-related behavior and different psychological status were investigated.

\section{Methods}

\section{Study design and sample}

We conducted an anonymous online health-related behavior and psychological survey of pregnant women from March 14 to April 7, following the peak of COVID-19 in China. We provided informed consent on the first page of the survey. The survey links or QR codes (both created through Wenjuanxing platform) were forwarded online to pregnant women living in mainland China through social media (WeChat). Pregnant women with single pregnancy and gestational age $\geq 4$ weeks who were willing to participate in the survey were included in this study. Respondents who returned incomplete survey or survey with contradictions between different items or in the same multiple-choice items were excluded.

\section{Measures}

Demographic data included age, education, monthly household income status, living cities during the last month (Wuhan, others), pre-pregnancy body mass index (BMI), gestational age, gravidity, parity and pregnancy complications. Other information included perception of the likelihood of infection, knowledge about COVID-19 and knowledge about maternal and child protection. Nine health-related behavior changes were developed by the research team which included preventive behaviors, prenatal check-up behaviors and help-seeking behaviors. 
The Generalized Anxiety Disorder scale (GAD-7) was used to measure anxiety symptoms of pregnant women. GAD-7 consists of 7 items which assess seven symptoms during the last two weeks [16]. Each item is scored from 0 to 3 , with a total score of 0-21 points. The cutoff for mild, moderate and severe anxiety were 5,10 and 15 , respectively [16]. Cronbach's a of GAD-7 in this study was 0.96 .

Edinburgh Postnatal Depression Scale (EPDS) was used to measure antenatal depressive symptoms of pregnant women. EPDS, originally used as a screening tool for postpartum depression, has also been shown to be validated for antenatal depression detection [17-19]. This self-rating scale consists of 10 items. Each item is rated on a 4-point scale ( $0-3$ points), with a total score of $0-30$ points. A cutoff of $\geq$ 10 was recommended for clinical depression in mainland China (sensitivity $80.00 \%$, specificity $83.03 \%$ ) [20]. Cronbach's a of EPDS in this study was 0.86 .

The stress of pregnant women was measured by Perceived Stress Scale (PSS). PSS contains 14 items which assess perceptions of stress during the last month [21]. Each item is rated on a 5-point scale (0-4 points), with a total score of $0-56$ points. The higher the score, the greater the perceived stress level. Chinese version PSS (CPSS) has good reliability (Cronbach's a coefficient 0.78 ) and validity, with a cutoff of $>25$ recommended to detect health risk stress based on urban residents survey [22]. Cronbach's a of CPSS in this study was 0.79 .

\section{Statistical analysis}

SPSS20.0 (IBM) was used for statistical analysis, and descriptive statistics included the means and standard deviations (SD) or median and inter-quartile range (IQR) (for continuous variables) and frequency and percentage (for categorical variables). Wilcoxon signed-rank test was used to compare health-related behavior changes before and during the COVID-19 outbreak for continuous variables with a non-normal distribution. To address our second research question, Spearman's rank correlation analysis was used to determine the relationship between continuous characteristics variables, continuous behavior variables and different psychological status, and logistic regression analysis were used to calculate the univariate associations between categorical characteristics variables, categorical behavior variables, and different psychological status. In the logistic regression analysis, associations were assessed by odds ratio (OR) and $95 \%$ confidence interval $(\mathrm{Cl}) . \mathrm{P}<0.05$ was statistically significant.

\section{Results}

\section{Characteristics of pregnant women}

A total of 155 questionnaires were collected and 42 questionnaires did not meet the inclusion and exclusion criteria (four pregnant women refused, six of the women were not single pregnancy, 11 women may not have been pregnant because the last menstrual period was close to the date of the survey and 21 women returned questionnaires with contradictions between different items or in the same multiplechoice items). The characteristics of 113 pregnant women were shown in Table 1. The pregnant women 
were mainly from the provinces and cities of Hubei, Henan, Hebei, Guangdong, Beijing and Tianjin. The mean age of pregnant women was $29.4 \pm 4.5$ years. The median perception of the likelihood of infection of pregnant women was 1.0 (IQR $0.0-2.0$ ). The percentage of pregnant women who had a lot, had somewhat and had little knowledge about COVID-19 were $49.6 \%, 47.8 \%$ and $2.7 \%$, respectively. In addition, the percentage of pregnant women who had a lot, had some-what and had little knowledge about maternal and child protection were $38.1 \%, 58.4 \%$ and $3.5 \%$, respectively. 
Table 1

Characteristics of pregnant women $(n=113)$

\section{Characteristics}

Education

Primary school or less

High school or technical secondary school

College or junior college

Master or above

Monthly household income before the outbreak

$<3000 ¥$

$3000-4999 ¥$

$5000-9999 ¥$

$\geq 10000 ¥$

Household income during the last month

No income

$<3000 ¥$

$3000-49997$

$5000-9999 ¥$

$\geq 10000 ¥$

Living cities during the last month

Wuhan

Others

Pre-pregnancy BMI $\left(\mathrm{Kg} / \mathrm{m}^{2}\right)$

$<18.5$

$18.5-23.9$

$\geq 24$

Gestational week

First trimester

a Included gestation diabetes, gestation hypertension, hyperemesis gravidarum, excess weight gain, threatened abortion, fetal malformation or cardiac dysplasia, others

Frequency

Percentage (\%)

$\begin{array}{ll}3 & 2.7\end{array}$

$\begin{array}{ll}7 & 6.2\end{array}$

$77 \quad 68.1$

$26 \quad 23.0$

$\begin{array}{ll}3 & 2.7\end{array}$

$12 \quad 10.6$

$26 \quad 23.0$

$\begin{array}{ll}72 & 63.7\end{array}$

$14 \quad 12.4$

980

$24 \quad 21.2$

$33 \quad 29.2$

$\begin{array}{ll}33 & 29.2\end{array}$

$24 \quad 21.2$

$\begin{array}{ll}89 & 78.8\end{array}$

$24 \quad 21.2$

$77 \quad 68.1$

$12 \quad 10.6$

20

17.7 


\begin{tabular}{|lll|}
\hline Characteristics & Frequency & Percentage (\%) \\
\hline Second trimester & 60 & 53.1 \\
\hline Third trimester & 33 & 29.2 \\
\hline Gravidity history & & \\
\hline First & 39 & 34.5 \\
$\geq 2$ & 74 & 65.5 \\
\hline Parity history & & \\
\hline Primiparous & 46 & 40.7 \\
\hline Multiparous & 67 & 59.3 \\
\hline Pregnancy complications ${ }^{a}$ & & \\
\hline Yes & 11 & 9.7 \\
\hline No & 102 & 90.3 \\
\hline $\begin{array}{l}\text { a Included gestation diabetes, gestation hypertension, hyperemesis gravidarum, excess weight gain, } \\
\text { threatened abortion, fetal malformation or cardiac dysplasia, others }\end{array}$ \\
\hline
\end{tabular}

\section{Health-related Behavior Changes Of Pregnant Women}

Following the peak of the COVID-19 epidemic, almost all pregnant women wore a mask when going out and changed location or tried to stay away from those who seem to have a cold or fever (cough, sneeze, etc.) on public transport or indoors. Most (96.5\%) pregnant women washed hands more often than before, especially after interacting with others outside. In addition, pregnant women washed hands with soap or hand sanitizer more frequently and went out less often than before the epidemic $(P<.001)$. Affected by the COVID-19 epidemic, more than a third (41.6\%) of pregnant women did not attend antenatal check-ups on time or did not attend antenatal check-ups. Many (77.9\%) pregnant women sought help when feeling panic or anxiety. The two most frequent sources of help were "medical staff $(53.1 \%)$ " and "relatives or friends (48.7\%)". Few pregnant women turned to psychological workers. (Table 2). 
Table 2

Health-related behavior changes of pregnant women $(n=113)$

\section{Behaviors}

\section{$\mathrm{N}$}

(\%)/Median

(IQR)

Wear a mask when going out

Yes

111

$(98.2 \%)$

No

$2(1.8 \%)$

Change location or try to stay away from those who seem to have a cold or fever (cough, sneeze, etc.) on public transport or indoors

Yes

112

$(99.1 \%)$

No

$1(0.9 \%)$

Wash hands more often than before, especially after interacting with others outside

Yes

No

$4(3.5 \%)$

Average daily wash hands with soap/hand sanitizer

One month before knowing the COVID-19 outbreak

$4.0(2.0-$

8.0)

During the last month

6.0 (3.5-

10.0)

Average weekly spit on the ground

One month before knowing the COVID-19 outbreak

$0(0.0-0.0)$

During the last month

$0(0.0-0.0)$

Average weekly go out

One month before knowing the COVID-19 outbreak

$7.0(5.0-$

7.0)

IQR: Inter-quartile range

a Wilcoxon signed-rank test 


\begin{tabular}{|l|l|}
\hline Behaviors & $\begin{array}{l}\text { N } \\
\text { (\%)/Median }\end{array}$ \\
\hline (IQR)
\end{tabular}

\section{Psychological Status Of Pregnant Women}

The psychological status (anxiety, depression, and stress) of pregnant women was shown in Table 3. We found that more than half of pregnant women had anxiety (64.6\%), depression (58.4\%) or health risk stress (54.9\%). 
Table 3

Psychological status of pregnant women $(n=113)$

\begin{tabular}{|lll|}
\hline Items & Frequency & Percentage (\%) \\
\hline Anxiety & & 35.4 \\
\hline No & 40 & 47.8 \\
\hline Mild & 54 & 12.4 \\
\hline Moderate & 14 & 4.4 \\
\hline Severe & 5 & 64.6 \\
Mild to severe & 73 & \\
\hline Depression & & 41.6 \\
\hline No & 47 & 58.4 \\
\hline Yes & 66 & \\
\hline Health risk stress & & 45.1 \\
\hline No & 51 & 54.9 \\
\hline Yes & 62 & \\
\hline GAD-7: Generalized Anxiety Disorder scale; EPDS: Edinburgh Postnatal Depression Scale; CPSS: \\
\hline Chinese version Perceived Stress Scale
\end{tabular}

\section{Relationship Between Pregnant Women's Characteristics And Different Psychological Status}

As shown in Table 4, perception of the likelihood of infection was significantly positively correlated with anxiety, depression and stress $(P<0.001)$. We found significant associations between the following variables in the logistic regression analysis: monthly household income before the outbreak, household income during the last month, pregnancy complications, knowledge about maternal and child protection and depression; monthly household income before the outbreak, gestational age, knowledge about COVID-19, knowledge about maternal and child protection and health risk stress $(P<0.05)($ Table 5$)$. 
Table 4

Correlation between continuous characteristics variables, continuous behavior variables, and different psychological status $(n=113)$

\begin{tabular}{|c|c|c|c|c|c|c|}
\hline \multirow[t]{2}{*}{ Items } & \multicolumn{2}{|c|}{ GAD-7 scores } & \multicolumn{2}{|c|}{ EPDS scores } & \multicolumn{2}{|c|}{ CPSS scores } \\
\hline & $r_{s}$ & $\mathbf{P}$ & $r_{s}$ & $\mathbf{P}$ & $\mathbf{r}_{\mathbf{s}}$ & $\mathbf{P}$ \\
\hline Age & -0.107 & 0.257 & -0.134 & 0.158 & -0.110 & 0.246 \\
\hline Perception of the likelihood of infection ${ }^{a}$ & 0.384 & 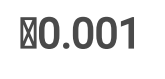 & 0.397 & $凶 0.001$ & 0.384 & $凶 0.001$ \\
\hline Behavior 1 & 0.131 & 0.165 & -0.033 & 0.727 & -0.049 & 0.610 \\
\hline Behavior 2 & 0.004 & 0.962 & 0.094 & 0.320 & 0.075 & 0.427 \\
\hline Behavior 3 & 0.190 & 0.043 & 0.097 & 0.309 & 0.269 & 0.004 \\
\hline Behavior 4 & -0.084 & 0.377 & -0.129 & 0.172 & 0.013 & 0.890 \\
\hline \multicolumn{7}{|c|}{$\begin{array}{l}\text { GAD-7:, Generalized Anxiety Disorder scale; EPDS:, Edinburgh Postnatal Depression Scale; CPSS: } \\
\text { Chinese version Perceived Stress Scale; } r_{s} \text { : Spearman's rank correlation coefficient }\end{array}$} \\
\hline \multicolumn{7}{|c|}{ Behavior 1: Average daily wash hands with soap or hand sanitizer during the last month } \\
\hline \multicolumn{7}{|c|}{ Behavior 2: Average weekly spit on the ground during the last month } \\
\hline \multicolumn{7}{|c|}{ Behavior 3: Average weekly go out during the last month } \\
\hline \multicolumn{7}{|c|}{ Behavior 4: Average weekly shower during the last month } \\
\hline
\end{tabular}


Table 5

Association between categorical characteristics variables, categorical behavior variables and different psychological status $(n=113)$

\begin{tabular}{|c|c|c|c|}
\hline \multirow[t]{2}{*}{ Items } & Anxiety & Depression & $\begin{array}{l}\text { Health Risk } \\
\text { Stress }\end{array}$ \\
\hline & OR(95\%Cl) & OR(95\%Cl) & OR(95\%Cl) \\
\hline \multicolumn{4}{|c|}{$\begin{array}{l}\text { Monthly household income before the } \\
\text { outbreak }\end{array}$} \\
\hline$\otimes 5000 ¥$ & 1.00 & 1.00 & 1.00 \\
\hline $5000-9999 ¥$ & $0.25(0.06-1.10)$ & $\begin{array}{l}0.13(0.02- \\
0.71)^{*}\end{array}$ & $\begin{array}{l}0.16(0.04- \\
0.69)^{\star}\end{array}$ \\
\hline$\geq 10000 ¥$ & $0.50(0.13-1.94)$ & $\begin{array}{l}0.20(0.04- \\
0.97)^{\star}\end{array}$ & $\begin{array}{l}0.31(0.08- \\
1.20)\end{array}$ \\
\hline
\end{tabular}

Household income during the last

month

$\begin{array}{llll}\varangle 5000 ¥ & 1.00 & 1.00 & 1.00 \\ 5000-9999 ¥ & 0.72(0.28-1.83) & 0.64(0.25-1.60) & 0.66(0.27- \\ & & & 1.62) \\ \geq 10000 ¥ & 0.82(0.32-2.10) & 0.39(0.16- & 0.58(0.24- \\ & & 0.98)^{\star} & 1.44)\end{array}$

Gestational age

First trimester

1.00

1.00

1.00

Second trimester

$0.80(0.27-2.38)$

$0.56(0.19-1.66)$

$0.20(0.05-$

$0.76)^{\star}$

Tird trimester

$0.66(0.20-2.15)$

$0.51(0.16-1.67)$

$0.11(0.03-$ $0.47)^{\star \star}$

Pregnancy complications

No

1.00

1.00

1.00

Yes

$1.52(0.38-6.08)$

$8.21(1.01-$

$66.56) *$

$1.50(0.41-$ 5.43)

Knowledge about COVID-19

OR: Odds ratio; Cl: Confidence interval. Education, cities during the last month, pre-pregnancy BMI $\left(\mathrm{Kg} / \mathrm{m}^{2}\right)$, gravidity and parity history, behavior of wearing a mask when going out, behavior of changing location or trying to stay away from those who seem to have a cold or fever (cough, sneeze, etc.) on public transport or indoors and behavior of washing hands more often than before, especially after interacting with others outside were not significantly associated with psychological status (anxiety,depression and health risk stress) $(P>0.05)$ and not shown in the table.

* $P<0.05 ; * \star P<0.01 ; * * * P<0.001$ 


\begin{tabular}{|c|c|c|c|}
\hline \multirow[t]{2}{*}{ Items } & Anxiety & Depression & $\begin{array}{l}\text { Health Risk } \\
\text { Stress }\end{array}$ \\
\hline & $\mathrm{OR}(95 \% \mathrm{Cl})$ & $\mathrm{OR}(95 \% \mathrm{Cl})$ & $\mathrm{OR}(95 \% \mathrm{Cl})$ \\
\hline Know a lot & 1.00 & 1.00 & 1.00 \\
\hline Some-what & $1.54(0.70-3.40)$ & $1.09(0.51-2.33)$ & $\begin{array}{l}2.67(1.23- \\
5.79)^{\star}\end{array}$ \\
\hline Know little & $0.32(0.03-3.79)$ & $\begin{array}{l}1.50(0.13- \\
17.52)\end{array}$ & $\begin{array}{l}2.67(0.23- \\
31.15)\end{array}$ \\
\hline \multicolumn{4}{|l|}{$\begin{array}{l}\text { Knowledge about maternal and child } \\
\text { protection }\end{array}$} \\
\hline Know a lot & 1.00 & 1.00 & 1.00 \\
\hline Some-what & $1.96(0.88-4.38)$ & $\begin{array}{l}3.19(1.43- \\
7.12)^{\star \star}\end{array}$ & $\begin{array}{l}3.15(1.42- \\
7.02)^{\star \star}\end{array}$ \\
\hline Know little & $0.79(0.10-6.15)$ & $\begin{array}{l}1.39(0.18- \\
10.80)\end{array}$ & $\begin{array}{l}5.06(0.48- \\
52.88)\end{array}$ \\
\hline \multicolumn{4}{|c|}{ Prenatal check-up during the COVID-19 outbreak } \\
\hline No prenatal check-up & 1.00 & 1.00 & 1.00 \\
\hline I had prenatal check-up on time & $2.67(0.82-8.64)$ & $\begin{array}{l}3.61(1.03- \\
12.72)^{\star}\end{array}$ & $\begin{array}{l}1.08(0.34- \\
3.48)\end{array}$ \\
\hline I were not on time for prenatal check-up & $\begin{array}{l}3.07(0.84- \\
11.17)\end{array}$ & $\begin{array}{l}5.75(1.45- \\
22.78)^{\star}\end{array}$ & $\begin{array}{l}0.63(0.18- \\
2.21)\end{array}$ \\
\hline \multicolumn{4}{|l|}{ Help-seeking behavior } \\
\hline No & 1.00 & 1.00 & 1.00 \\
\hline Yes & $\begin{array}{l}7.71(2.85- \\
20.92)^{\star \star \star}\end{array}$ & $\begin{array}{l}4.11(1.59- \\
10.61)^{\star *}\end{array}$ & $\begin{array}{l}2.17(0.88- \\
5.36)\end{array}$ \\
\hline \multicolumn{4}{|c|}{$\begin{array}{l}\text { OR: Odds ratio; Cl: Confidence interval. Education, cities during the last month, pre-pregnancy BMI } \\
\left(\mathrm{Kg} / \mathrm{m}^{2}\right) \text {, gravidity and parity history, behavior of wearing a mask when going out, behavior of } \\
\text { changing location or trying to stay away from those who seem to have a cold or fever (cough, sneeze, } \\
\text { etc.) on public transport or indoors and behavior of washing hands more often than before, especially } \\
\text { after interacting with others outside were not significantly associated with psychological status } \\
\text { (anxiety,depression and health risk stress) }(\mathrm{P}>0.05) \text { and not shown in the table. }\end{array}$} \\
\hline \multicolumn{4}{|l|}{ * $P<0.05 ; * \star P<0.01 ; * \star * P<0.001$} \\
\hline
\end{tabular}

\section{Relationship Between Health-related Behavior And Different Psychological Status}


The frequency of going out was significantly positively correlated with anxiety and stress $(P=0.043, P=$ 0.004 , respectively) (Table 4). Significant associations were discovered between prenatal check-ups behavior and depression as well as between help-seeking behavior and anxiety and depression symptoms (Table 5).

\section{Discussion}

This study describes the health-related behavior changes and psychological status of pregnant women after the peak of COVID-19 outbreak in China. An earlier study in China found that most pregnant women took protective measures including wearing masks, washing hands frequently and staying at home [12]. Our study got similar results. In addition, pregnant women washed hands with soap or hand sanitizer more frequently and went out less often than before the epidemic. However, there was no significant change in the frequency of spitting on the ground and bathing during the COVID-19 outbreak compared with before the outbreak. The possible reason is that most (92.9\%) pregnant women did not spit on the ground. Therefore, the frequency of spitting on the ground didn't decline significantly. As for bathing, as a habitual behavior, it's hard to change [23]. Another reason may be that we had assumed that pregnant women would change clothes and shower after contact with others. In this study, the frequency of pregnant women going out every week was low during the outbreak (median 2.0, IQR 0.0-4.0). This had little influence on the frequency of bathing among pregnant women who bathed almost every day.

Earlier studies reported that pregnant women from different countries experienced different levels of anxiety or depression during the early or peak period of COVID-19 [5-8, 12]. In Wuhan, 24.5\% of pregnant women reported self-rated anxiety, compared with $10.4 \%$ of that in Chongqing, China [6]. The rate of anxiety (assessed by state-trait anxiety inventory) was $38.2 \%$ among pregnant women in Italy [8]. The rate of depression (assessed by EPDS) was $35.4 \%$ among Turkish pregnant women and $29.6 \%$ among Chinese women in the third trimester of pregnancy $[5,7]$. Although the peak of the epidemic in China has passed, the psychological condition of pregnant women does not seem to improve, as we found that 73 (64.6\%), 66 (58.4\%) and 62 (54.9\%) pregnant women had anxiety, depression and health risk stress, respectively. This may be due to the uncertainty of the disease in the absence of an effective vaccine. The news of domestic sporadic cases and imported cases from abroad may cause fear and panic among pregnant women.

Previous study found higher rates of anxiety among pregnant women in Wuhan than Chongqing during the COVID-19 outbreak [6]. However, we found no significant association between cities (Wuhan and other cities) and different psychological status (anxiety, depression and health risk stress). The possible reason is that the COVID-19 outbreak in Wuhan was under control. Pregnant women in Wuhan were not as feared and scared as they used to be. Another reason is the presence of imported cases in other provinces and cities such as Beijing, Guangdong and Tianjing in China. This can cause some anxiety, fear and panic in the absence of a well-organized approach to preventing and controlling imports from abroad at beginning. Due to the publicity of the government and the media, only three $(2.7 \%)$ pregnant women had little knowledge about COVID-19, and four (3.5\%) had little knowledge about maternal and child 
protection. This suggests that pregnant women were well informed about COVID-19 and maternal and child protection. We found that pregnant women who knew some-what about COVID-19 were more likely to have health risk stress than those who had a lot of knowledge. Pregnant women who knew some-what about maternal and child protection were more likely to develop depression and health risk stress than those who knew a lot. In addition, pregnant women with higher anxiety, depression or stress perceived a higher likelihood of infection. So pregnant women's understanding of COVID-19 and maternal and child protection and their perception of the likelihood of infection should be considered in psychological intervention. We also need to pay attention to specific demographic characteristics (household income, gestational age and pregnancy complications) of pregnant women, as they can affect psychology.

A study from Hong Kong found that general population who felt emotionally distressed were more likely to avoid going out than others in the initial stage of H1N1 outbreak [24]. However, we found that the frequency of going out was positively correlated with anxiety and stress. One possible explanation is that pregnant women with higher anxiety and stress tended to go out to relax after staying at home for a long time. Another possibility is that we observed an opposite relationship, where the more often they went out, the more pregnant women worried about getting infected, leading to higher anxiety and stress. More research is needed to clarify the relationship between health-related behavior and psychology among pregnant women during the COVID-19 outbreak. Pregnant women who had antenatal check-ups were more likely to be depressed than those who had not. This is probably because pregnant women were worried about getting infected during antenatal visits [25]. A study in Hong Kong found that $66.7 \%$ pregnant women feared going to the hospital for a prenatal check-up during the 2003 severe acute respiratory syndrome (SARS) outbreak [26]. As a result, in our study, some (12.4\%) pregnant women did not have antenatal visits and some (29.2\%) pregnant women did not have them on time. It's normal that 88 (77.9\%) pregnant women sought help when they felt panic and anxiety during the COVID-19 outbreak. However, those who sought help were more likely to be anxious and depressed than those who did not. The possible explanation is that few pregnant women sought help from psychological workers in our study (those who can provide professional psychological screening and intervention), left unresolved psychological problems after help seeking. Another possible explanation is that pregnant women may be more concerned about how to protect themselves and their babies rather than psychological problems in the process of seeking help. As a result, the psychological status of pregnant women has not received good attention. Therefore, screening for anxiety, depression and stress and professional psychological interventions whether online or offline are needed.

There are several limitations to this study. First, we did not examine a history of mental health problems in pregnant women, which may influence anxiety, depression and stress scores. Second, this study was a cross-sectional study in nature, which makes it impossible to determine the causal relationship between some variables. Finally, we did not conduct multivariate logistic regression analysis to adjust for confounders because the sample of outcome variables was small and the relationships between some variables were so complex to determine. The results of this study should be interpreted with caution due to false-positive or false-negative results from small sample and univariate logistic regression. More 
research is needed to establish structural equation models to determine the path relationship between pregnant women's characteristics, health-related behavior and psychology.

\section{Conclusions}

Preventive measures were adopted by most pregnant women and the behavior of "washing hands" and "going out" significantly changed compared with before the outbreak. More than a third of pregnant women cancelled or delayed antenatal visits. Most pregnant women were willing to seek help when feeling panic and anxiety. However, few pregnant women turned to psychological workers. Although the peak epidemic had passed in China, psychological distress of the pregnant woman did not ease. We found several factors were associated with different psychological status. Psychological intervention should be developed according to the characteristics of pregnant women, such as household income, gestational age, pregnancy complications and perception of the likelihood of infection. Knowledge about COVID-19 and maternal and child protection should be shared as much as possible with pregnant women. Based on the results of relationship between health-related behavior and different psychological status, greater attention needs to be paid to the mental health of pregnant women who undergo antenatal care and seek help, and screening for mental health problems is suggested.

\section{Abbreviations}

COVID-19

Coronavirus disease 2019; BMI:Body mass index; GAD-7:Generalized Anxiety Disorder scale; EPDS:Edinburgh Postnatal Depression Scale; PPS:Perceived Stress Scale; CPSS:Chinese version PPS; SD:Standard deviations; IQR:Inter-quartile range; OR:Odds ratio; Cl:Confidence interval; SARS:Severe acute respiratory syndrome

\section{Declarations}

\section{Acknowledgements}

Not applicable.

\section{Author's contributions}

RXT, XLC, CD, YQL and ZJZ contributed to the study design. XLC and CD contributed to the data collection and management. RXT and XZ contributed to the data collection, handling and analysis. RXT drafted the manuscript and $X Z$ proofread the manuscript. YQL and ZJZ contributed to the data collection and revised the manuscript. All authors read and approved the final manuscript.

\section{Funding}

There was no funding for this study. 
Availability of data and materials

The datasets used and analyzed during the current study are available from the corresponding author on reasonable request.

\section{Ethics approval and consent to participate}

The study was approved by the Medical Ethics Committee of Wuhan University School of Medical (WHU2020-YF0046). An electronic informed consent was obtained from all participants before completing the questionnaire.

\section{Consent for publication}

It is not applicable.

\section{Competing interests}

All authors declare that they have no competing interests.

\section{References}

1. Nishiura H, Jung SM, Linton NM, Kinoshita R, Yang $Y$, Hayashi $K$, et al. The extent of transmission of novel coronavirus in Wuhan, China, 2020. J Clin Med. 2020;9(2):330.

2. Rasmussen SA, Smulian JC, Lednicky JA, Wen TS, Jamieson DJ. Coronavirus Disease 2019 (COVID19) and Pregnancy: What obstetricians need to know. Am J Obstet Gynecol. 2020;222(5):415-26.

3. China daily. Key phrases in China's COVID-19 fight. 2020. https://covid19.chinadaily.com.cn/a/202004/21/WS5e9e9f83a3105d50a3d17c161.html. Accessed 14 Jun 2020.

4. Ecns.cn. Full Text: Fighting COVID-19: China in Action. 2020. http://www.ecns.cn/news/politics/2020-06-07/detail-ifzwzeax1201671.shtml.

5. Accessed 14 Jun 2020.

6. Wu Y, Zhang C, Liu H, Duan C, Li C, Fan J, et al. Perinatal depressive and anxiety symptoms of pregnant women during the coronavirus disease 2019 outbreak in China. Am J Obstet Gynecol. 2020;223(2):240-1.

7. Liu X, Chen M, Wang Y, Sun L, Zhang J, Shi Y, et al. Prenatal anxiety and obstetric decisions among pregnant women in Wuhan and Chongqing during the COVID-19 outbreak: a cross-sectional study. BJOG. 2020. doi:10.1111/1471-0528.16381.

8. Durankuş F, Aksu E. Effects of the COVID-19 pandemic on anxiety and depressive symptoms in pregnant women: a preliminary study. J Matern Fetal Neonatal Med. 2020:1-7. doi:10.1080/14767058.2020.1763946.

9. Mappa I, Distefano FA, Rizzo G. Effects of coronavirus 19 pandemic on maternal anxiety during pregnancy: a prospectic observational study. J Perinat Med. 2020;48(6):545-50. 
10. Berthelot N, Lemieux R, Garon Bissonnette J, Drouin Maziade C, Martel É, Maziade M. Uptrend in distress and psychiatric symptomatology in pregnant women during the coronavirus disease 2019 pandemic. Acta Obstet Gynecol Scand. 2020;99(7):848-55.

11. Taubman Ben-Ari O, Chasson M, Abu Sharkia S, Weiss E. Distress and anxiety associated with COVID-19 among Jewish and Arab pregnant women in Israel. J Reprod Infant Psychol. 2020;38(3):340-8.

12. Corbett GA, Milne SJ, Hehir MP, Lindow SW, O Connell MP. Health anxiety and behavioural changes of pregnant women during the COVID-19 pandemic. Eur J Obstet Gynecol Reprod Biol. 2020;249:96-7.

13. Yue C, Liu C, Wang J, Zhang M, Wu H, Li C, et al. Association between social support and anxiety among pregnant women in the third trimester during the coronavirus disease 2019 (COVID-19) epidemic in Qingdao, China: The mediating effect of risk perception. Int J Soc Psychiatry. 2020:20764020941567. doi:10.1177/0020764020941567.

14. Nwafor JI, Aniukwu JK, Anozie BO, Ikeotuonye AC, Okedo Alex IN. Pregnant women's knowledge and practice of preventive measures against COVID-19 in a low-resource African setting. Int J Gynaecol Obstet. 2020;150(1):121-3.

15. Liu X, Luo W, Li Y, Li C, Hong Z, Chen H, et al. Psychological status and behavior changes of the public during the COVID-19 epidemic in China. Infect Dis Poverty. 2020;9(1):58.

16. Thapa SB, Mainali A, Schwank SE, Acharya G. Maternal mental health in the time of the COVID-19 pandemic. Acta Obstet Gynecol Scand. 2020;99(7):817-8.

17. Spitzer RL, Kroenke K, Williams JBW, Löwe B. A Brief Measure for Assessing Generalized Anxiety Disorder. Arch Intern Med. 2006;166(10):1092-7.

18. Cox JL, Holden JM, Sagovsky R. Detection of postnatal depression. Development of the 10-item Edinburgh Postnatal Depression Scale. Br J Psychiatry. 1987;150:782-6.

19. Joshi U, Lyngdoh T, Shidhaye R. Validation of hindi version of Edinburgh postnatal depression scale as a screening tool for antenatal depression. Asian J Psychiatr. 2020;48:101919.

20. Vázquez MB, Míguez MC. Validation of the Edinburgh postnatal depression scale as a screening tool for depression in Spanish pregnant women. J Affect Disord. 2019;246:515-21.

21. Wang Y, Guo X, Lau Y, Chan KS, Yin L, Chen J. Psychometric evaluation of the Mainland Chinese version of the Edinburgh Postnatal Depression Scale. Int J Nurs Stud. 2009;46(6):813-23.

22. Cohen S, Kamarck T, Mermelstein R. A Global Measure of Perceived Stress. J Health Soc Behav. 1983;24(4):385-96.

23. Yang TZ, Huang HT. An epidemiological study on stress among urban residents in social transition period(in Chinese). Zhonghua Liu Xing Bing Xue Za Zhi. 2003;24:760-4.

24. Cowling BJ, Ng DMW, Ip DKM, Liao Q, Lam WWT, Wu JT, et al. Community Psychological and Behavioral Responses through the First Wave of the 2009 Influenza A(H1N1) Pandemic in Hong Kong. J Infect Dis. 2010;202(6):867-76. 
25. Lau JT, Griffiths S, Choi KC, Tsui HY. Avoidance behaviors and negative psychological responses in the general population in the initial stage of the H1N1 pandemic in Hong Kong. BMC Infect Dis. 2010;10(1):139.

26. Zeng LN, Chen LG, Yang CM, Zeng LP, Zhang LY, Peng TM. Mental health care for pregnant women in the COVID-19 outbreak is urgently needed. Women Birth. 2020. doi:10.1016/j.wombi.2020.03.009.

27. Lee DTS, Sahota D, Leung TN, Yip ASK, Lee FFY, Chung TKH. Psychological responses of pregnant women to an infectious outbreak: A case-control study of the 2003 SARS outbreak in Hong Kong. $J$ Psychosom Res. 2006;61(5):707-13. 